\title{
Additional Notes on the Reproduction of the Formosan Grass Lizard, Takydromus formosanus (Boulenger 1894) (Squamata: Lacertidae), from Southwestern Taiwan
}

\author{
Gerrut Norval ${ }^{1}$, Jean-Jay $\mathrm{Mao}^{2}$, Stephen R. Goldberg ${ }^{3}$, and Shao-Chang Huang ${ }^{4}$
}

${ }^{1}$ Applied Behavioural Ecology \& Ecosystem Research Unit, Department of Environmental Sciences, UNISA, Private Bag X6, Florida, 1710, Republic of South Africa (gnorval@gmail.com)

${ }^{2}$ Department of Forestry and Natural Resources, National Ilan University, No. 1, Sec. 1, Shen-Lung Rd., Yilan, 260, Taiwan, Republic of China ${ }^{3}$ Department of Biology, Whittier College, Whittier, California 90608, USA

${ }^{4}$ Queensland Brain Institute, QBI Building (79), Upland Road, The University of Queensland, St Lucia, QLD 4072, Australia

Photographs by the senior author.

Abstract.-We collected 13 (male = 2; female = 10; juvenile =1) Takydromus formosanus on an ad hoc basis from a Betelnut Palm (Areca catechu) plantation in Santzepu, Sheishan District, Chiayi County, as part of a herpetofaunal survey of the area. The smallest reproductively active female had a snout-vent length (SVL) of $44 \mathrm{~mm}$, and the smallest male with an enlarged tail base had a SVL of $41 \mathrm{~mm}$. A histological examination and oviposition indicated that the female reproductive cycle commences in April and lasts until at least June. We recorded no clutch sizes greater than two eggs. Takydromus formosanus is a poorly studied species, and many aspects of the reproductive biology of this species merit further research.

I

nformation on the reproductive patterns of reptiles and amphibians is critical to species conservation efforts (Gibbons 1994), so understanding, for example, the period of sperm production, timing of yolk deposition, and number and sizes of clutches produced is important. Even chance observations and studies generating limited data can provide important information, can be helpful in generating questions leading to comprehensive studies, or highlight aspects that warrant in-depth research. For that reason, the publication of such data should be encouraged.

The Formosan Grass Lizard (Takydromus formosanus [Boulenger 1894]; Fig.1) is endemic to the main island of Taiwan, where it was formerly believed to be the most widely distributed Takydromus species in Taiwan (Lue et al. 2002; Shang and Lin 2001). As for many other species, some confusion exists regarding the taxonomic history of $T$. formosanus. Until it was validated as a legitimate species, Stejneger's Grass Lizard (T. stejnegeri Van Denburgh 1912) was considered a synonym of T. formosanus (Boulenger 1917). More recently, a study (Lin 2003; Lin et al. 2002) using molecular markers indicated that the $T$. formosanus complex contained two other cryptic species from northern and eastern Taiwan; sub- sequently, Lue and Lin (2008) described T. viridipunctatus (the northern clade) and T. luyeanus (the eastern clade).

In a previous report, we described three clutches of Takydromus formosanus from southwestern Taiwan (Norval et al. 2007). In this report, we incorporate additional information concerning reproduction with our previous observations to provide a more comprehensive description of reproduction in T. formosanus from southwestern Taiwan. We also highlight those aspects that merit further research.

\section{Methods}

From early January 2004 to late October 2006, we collected T. formosanus on an ad hoc basis by hand or pitfall traps inside enclosures erected in a Betelnut Palm (Areca catechu) plantation in Santzepu, Sheishan District, Chiayi County (N2325'41”, E120²8'11”; WGS84; Norval et al. 2007).

For all collected lizards, we measured snout-vent length (SVL) and tail length (TL) with a transparent plastic ruler to the nearest $\mathrm{mm}$, scored the tail as complete or broken, and weighed the lizard to the nearest $0.1 \mathrm{~g}$ with a digital scale. If the lizard had suffered tail-loss in the past, the regenerated portion of the tail was also measured with a transparent plas- 


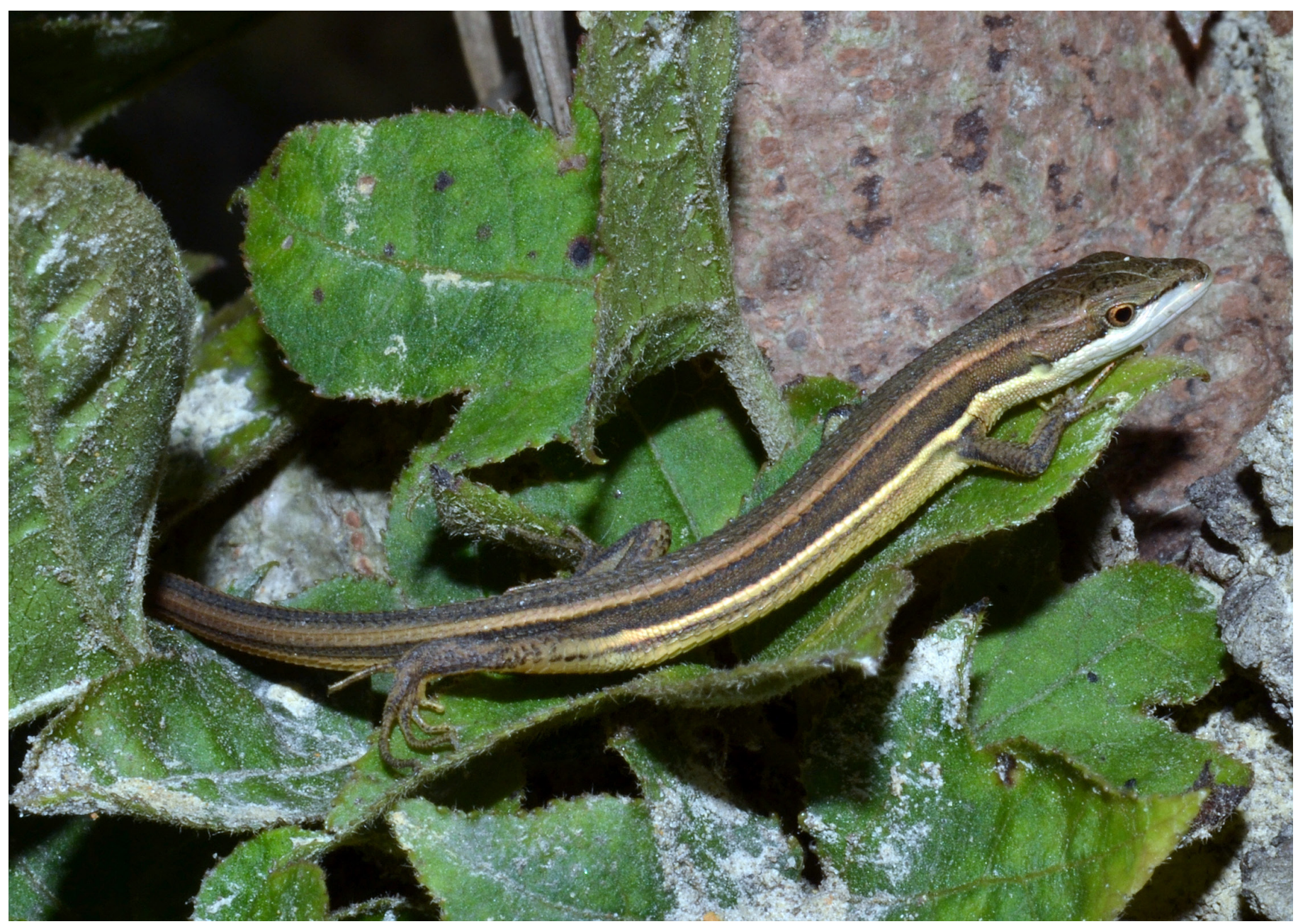

Fig. 1. The Formosan Grass Lizard (Takydromus formosanus) is a small diurnal species that lives on and among herbaceous vegetation at altitudes below $1,500 \mathrm{~m}$ on the main island of Taiwan.

tic ruler to the nearest $\mathrm{mm}$. In order to avoid injury to live lizards or induce tail-loss, no attempts were made to determine the sex of the lizards, other than visually examining the tail bases for the presence of enlarged hemipenal pouches.

Four $T$. formosanus died in pitfall traps and were subsequently deposited in the herpetological collection of the Natural History Museum of Los Angeles County (LACM), Los Angeles, California. These specimens were examined histologically to determine their reproductive state. The right gonad of each lizard was removed, dehydrated in an ascending series of ethanol, embedded in paraffin, sectioned at 5 $\mu$, mounted on glass slides, and stained with Harris' hematoxylin followed by an eosin counterstain. We examined the left ovaries of the four LACM specimens visually in situ or microscopically, and assigned them to one of four stages of the ovarian cycle: (1) inactive (no yolk deposition); (2) yolk deposition in one or more ovarian follicles; (3) oviductal eggs present and yolk deposition in one or more ovarian follicles; or (4) oviductal eggs present, and no yolk deposition in ovarian follicles.

\section{Results}

During the study period, we collected $13 T$. formosanus. Two of the collected lizards were positively sexed as males by the presence of enlarged tail bases (Fig. 2). One had SVL, TL, and body mass of $43 \mathrm{~mm}, 71 \mathrm{~mm}$, and $1.4 \mathrm{~g}$, the other 41 $\mathrm{mm}, 119 \mathrm{~mm}$, and $1.5 \mathrm{~g}$, respectively. Since a male with a SVL of $41 \mathrm{~mm}$ was found with an enlarged tail base, any lizard with a SVL $\geq 41 \mathrm{~mm}$ without an enlarged tail base was regarded as female. For females $(\mathrm{n}=10)$ SVL, TL, and body mass were $41-47 \mathrm{~mm}$ (mean $\pm \mathrm{SD}=45.3 \pm 1.5 \mathrm{~mm}$ ), $75-137 \mathrm{~mm}(118.4 \pm 16.7 \mathrm{~mm})$, and $1.2-1.8 \mathrm{~g}(1.5 \pm 0.2$ g), respectively. The smallest reproductively active female measured $44 \mathrm{~mm} \mathrm{SVL}$, and for reproducing females $(\mathrm{n}=5)$, SVL, TL, and body mass were 44-47 mm $(45.5 \pm 1.3 \mathrm{~mm})$, $96-137 \mathrm{~mm}(115.5 \pm 21.5 \mathrm{~mm})$, and $1.3-1.8 \mathrm{~g}(1.6 \pm 0.2 \mathrm{~g})$, respectively. The remaining lizard had a SVL, TL, and body mass of $30 \mathrm{~mm}, 55 \mathrm{~mm}$, and $0.5 \mathrm{~g}$, and was thus regarded as a juvenile that could not be accurately sexed.

Of the four specimens that were examined histologically, only two were reproductively active. A female collected in 
Table 1. A summary of the morphological data of gravid female Formosan Grass Lizards (Takydromus formosanus) from Santzepu and their eggs. ${ }^{1}=$ current study; ${ }^{2}=$ Norval et al. 2007; SVL = snout-vent length in $\mathrm{mm} ; \mathrm{BM}=$ body mass in g; CS = clutch size; $\mathrm{MEL}=$ mean egg length in $\mathrm{mm}(\mathrm{SD}) ; \mathrm{MEW}=$ mean egg width in $\mathrm{mm}(\mathrm{SD}) ; \mathrm{MEM}=$ mean egg mass in $\mathrm{g}(\mathrm{SD})$; $\mathrm{RCM}=$ relative clutch mass; ${ }^{*}=$ post-oviposition body mass; ${ }^{* *}=$ oviposition date; ${ }^{* * *}=$ collection date .

\begin{tabular}{|c|c|c|c|c|c|c|c|}
\hline Date & SVL & $\mathrm{BM}$ & CS & MEL & MEW & MEM & $\mathrm{RCM}$ \\
\hline Mar $2004^{* * * 1}$ & 45 & 1.8 & 2 & - & - & - & - \\
\hline Mar 2006***1 & 44 & 1.6 & 2 & - & - & - & - \\
\hline 22 Apr $2006^{* * 2}$ & 44 & $1.3^{*}$ & 2 & $8.2(0.04)$ & $5.15(0.14)$ & $0.1(0)$ & 15.39 \\
\hline 15 May $2006^{* * 2}$ & 46 & $1.5^{*}$ & 2 & $8.7(0.28)$ & $5.58(0.67)$ & $0.1(0)$ & 13.33 \\
\hline 26 Jun $2006^{* * 2}$ & 47 & $1.5^{*}$ & 2 & $8.73(0.11)$ & $5.43(0.04)$ & $0.1(0)$ & 13.33 \\
\hline
\end{tabular}

March 2004 (LACM 180394; SVL = $45 \mathrm{~mm}$; TL = $131 \mathrm{~mm}$; mass $=1.8 \mathrm{~g}$ ) had two enlarged ovarian follicles, $3 \mathrm{~mm}$ each, but no oviductal eggs. Another female, collected in March $2006(\mathrm{LACM} 180393 ; \mathrm{SVL}=44 \mathrm{~mm} ; \mathrm{TL}=75 \mathrm{~mm}$; mass $=$ $1.6 \mathrm{~g}$ ), had two oviductal eggs, and no yolk deposition in any ovarian follicles.

\section{Discussion}

Although Shang and Lin (2001) and Lue et al. (2002) made some references to reproduction in $T$. formosanus, no descriptions of sexually mature sizes were given, so no comparisons can be made. The smallest reproducing females $(\mathrm{n}=2)$ recorded in this study had a SVL of $44 \mathrm{~mm}$ (Table 1), and since a fairly large female $(S V L=41 \mathrm{~mm})$ from our study was still non-reproductive even though she was collected in July, female $T$. formosanus apparently become sexually mature at a SVL of ca. $44 \mathrm{~mm}$. In studies of T. hsuehshanensis and T. sauteri, Huang $(1998,2006)$ found that males become sexually mature at a slightly lesser SVL than females. Consequently, the sexes of $T$. formosanus become sexually mature at the sizes reported herein. Still, due to the very small sample sizes, additional research is needed for confirmation.

All of the reproductively active $T$. formosanus females described herein had a clutch size of two eggs, which is similar to the mean clutch size reported by Huang (2006). So, our data could not confirm the clutch sizes of two to six eggs reported by Lue et al. (1987) and Lue et al. (2002). Interestingly, T. viridipunctatus and $T$. luyeanus, which were formerly confused with $T$. formosanus, also have mean clutch sizes of two eggs, although a few females produce three eggs per clutch (Lue and Lin 2008). Consequently, we believe that clutches of at least three eggs are possible in some very large T. formosanus females, and the four to six eggs per clutch reported by Lue et al. (1987) could in fact be the result of counting oviductal eggs and enlarged ovarian follicles as a single clutch.

Studies involving T. septentrionalis (Ji et al. 2007; Luo et al. 2010), T. stejnegeri (Cheng and Lin 1987; Lin et al.
2004), and T. tachydromoides (Telford 1969) have indicated that these lizards are income breeders (i.e., they expend some acquired energy for reproduction soon after that energy is obtained; Jönsson 1997). Also, since multiple clutches of eggs in the same year have been reported for T. hsuehshanensis (Huang 1998), T. kuehnei (Norval and Mao 2008), T. luyeanus (Lue and Lin 2008), T. sauteri (Huang, 2006), T. septentrionalis (Ji et al. 2007; Luo et al. 2010), T. stejnegeri (Cheng and Lin 1987; Lin et al. 2004), T. tachydromoides (Telford 1969), and T. viridipunctatus (Lue and Lin 2008), this trait appears very common in the genus Takydromus. So,

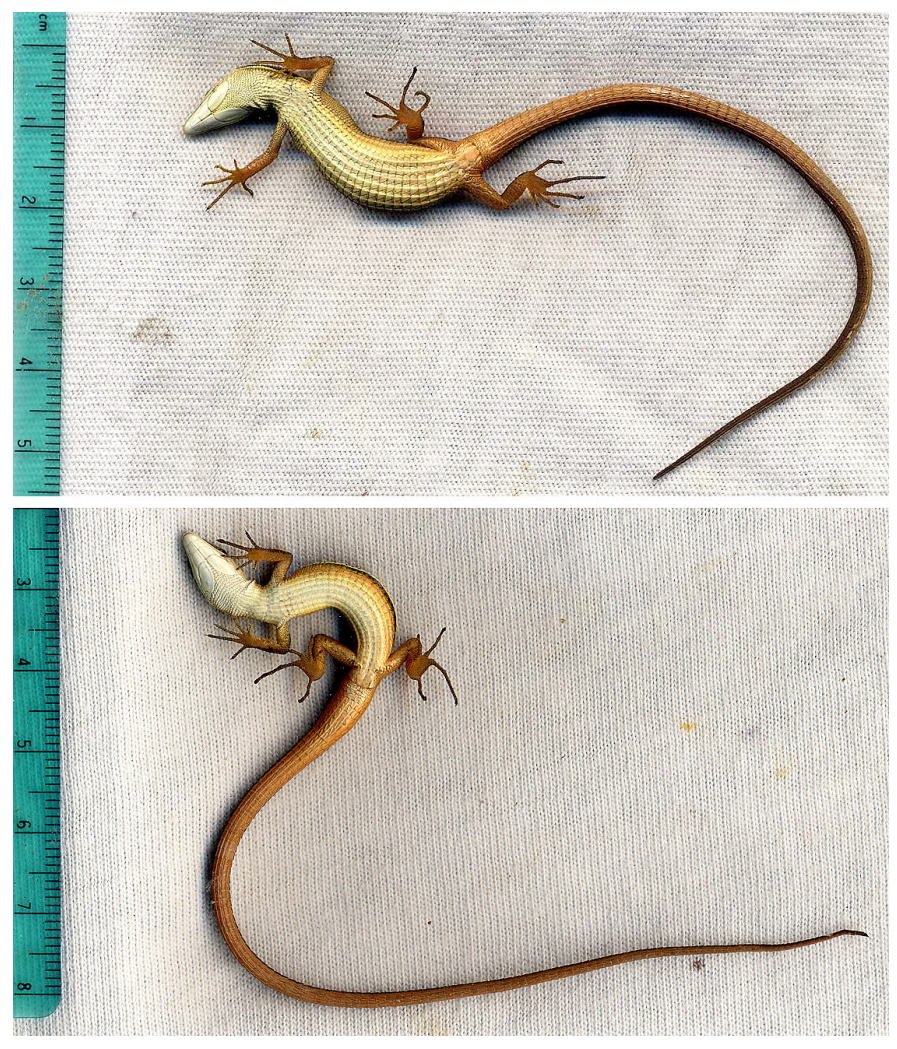

Fig. 2. Scanned images of the venters of a female Takydromus formosanus $(\mathrm{SVL}=46 \mathrm{~mm}$; top) and a male $(\mathrm{SVL}=41 \mathrm{~mm}$; bottom) from this study to illustrate the enlarged tail base of the male. 
even though we found no evidence that $T$. formosanus females produce multiple clutches in the same year, we suggest that producing more than one clutch of eggs per year is likely under suitable conditions.

Apart from the hatchlings described by Norval et al. (2007), we found no other descriptions. Since inter-population offspring size variations can exist within the same species (e.g., Du et al. 2010), we suggest that attempts should be made to obtain additional descriptions of hatchling $T$. formosanus as well as of all other species in the genus Takydromus.

Temperature during incubation can affect the fitness of hatchlings (Du and Ji 2006) and the incubation period (Birchard 2004; Chen et al. 2010). As with hatchling descriptions, the only record of an incubation period that we could find was that of Norval et al. (2007). Again, we advocate additional research on the incubation period of $T$. formosanus under natural conditions.

Due to the very small number of $T$. formosanus used in this study, we were unable to provide a complete description of the reproductive cycle of these lizards. However, Huang (2006) found that female $T$. sauteri become reproductively active as early as February, females with oviductal eggs are first apparent in April, and reproduction ceased in August. Similarly, Cheng and Lin (1987) determined that female $T$. stejnegeri commenced reproduction in March and ceased in August, whereas female T. hsuehshanensis are reproductive from April to August (Huang 1998). As for T. luyeanus and $T$. viridipunctatus, which were formerly confused with $T$. formosanus, Lue and Lin (2008) stated that gravid females of the former can be found from May to August, while gravid females of the latter can be found from April to August. Based on the findings of this study, we conclude that the reproductive season of female $T$. formosanus is most likely very similar to that of congeners in Taiwan (i.e., spring and summer; Table 1). We suggest that a detailed empirical study be implemented to determine the exact extent of the reproductive cycle of $T$. formosanus of both sexes in Taiwan. Because of conservation concerns, we discourage the collection of animals from the wild for such a study, and suggest that existing museum specimens be utilized when possible.

\section{Acknowledgements}

We express our gratitude to Christine Thacker of the Natural History Museum of Los Angeles County (LACM), Los Angeles, California, for allowing us to examine specimens LACM 180395, LACM 180397, LACM 180394, and LACM 180393.

\section{Literature Cited}

Birchard, G.F. 2004. Effects of incubation temperature. pp. 103-123. In: D.C. Deeming (ed.), Reptilian Incubation: Environment, Evolution, and Behaviour. Nottingham University Press, Nottingham, UK.

Boulenger, G.A. 1917. A revision of the genus Tachydromus. Memoirs of the Asiatic Society of Bengal 5: 207-235.

Chen, Y.H., S.P. Huang, M.H. Chang, and M.C. Tu. 2010. Thermal effects on embryogenesis and hatchlings of the grass lizard Takydromus stejnegeri (Squamata: Lacertidae) and implications of their potential for limiting its altitudinal distribution in Taiwan. Zoological Studies 49: 374-380.

Cheng, H.Y. and J.Y. Lin. 1987. Annual ovarian, fat body and liver cycles of the grass lizard Takydromus stejnegeri in Taiwan. Zoological Science 4: 557-561.

Du, W.G. and X. Ji. 2006. Effects of constant and fluctuating temperatures on egg survival and hatchling traits in the Northern Grass Lizard (Takydromus septentrionalis, Lacertidae). Journal of Experimental Zoology 305A: 47-54.

Du, W.G., X. Ji, Y.P. Zhang, Z.H. Lin, and X.F. Xu. 2010. Geographic variation in offspring size of a widespread lizard (Takydromus septentrionalis): Importance of maternal investment. Biological Journal of the Linnean Society 101: 59-67.

Gibbons, J.W. 1994. Reproductive patterns of reptiles and amphibians: Considerations for captive breeding and conservation, pp. 119-123. In: J.B. Murphy, K. Adler, and J.T. Collins (eds.), Captive Management and Conservation of Amphibians and Reptiles. Society for the Study of Amphibians and Reptiles, Ithaca, New York.

Huang, W.S. 1998. Reproductive cycle of the grass lizard, Takydromus hsuehshanensis, with comments on reproductive patterns of lizards from central high elevation areas of Taiwan. Copeia 1998: 866-873.

Huang, W.S. 2006. Ecology and reproductive patterns of the grass lizard, Takydromus sauteri, in a tropical rainforest of an East Asian island. Journal of Herpetology 40: 267-273.

Ji, X., W.G. Du, Z.H. Lin, and L.G. Luo. 2007. Measuring temporal variation in reproductive output reveals optimal resource allocation to reproduction in the Northern Grass Lizard, Takydromus septentrionalis. Biological Journal of the Linnean Society 91: 315-324.

Jönsson, K.I. 1997. Capital and income breeding as alternative tactics of resource use in reproduction. Oikos 78: 57-66.

Lin, C.F., Y.L. Chen, and Y.F. Tsai. 2004. A production of four successive clutches of eggs by a female grass lizard (Takydromus stejnegeri van Denburgh) in captivity. Endemic Species Research 6: 35-40.

Lin, S.M. 2003. Phylogeny and phylogeographic studies of Takydromus in Taiwan and adjacent regions (Squamata: Lacertidae). Ph.D. Dissertation, Department of Biology, National Taiwan Normal University, Taipei, Taiwan.

Lin, S.M., C.A. Chen, and K.Y. Lue. 2002. Molecular phylogeny and biogeography of the grass lizards genus Takydromus (Reptilia: Lacertidae) of East Asia. Molecular Phylogenetics and Evolution 22: 276-288.

Lue, K.Y. and S.M. Lin. 2008. Two new cryptic species of Takydromus (Squamata: Lacertidae) from Taiwan. Herpetologica 64: 276-288.

Lue, K.Y., S.H. Chen, Y.S. Chen, and S.L. Chen. 1987. Taiwan Lizards. Taiwan Provincial Department of Education, Taipei (in Chinese).

Lue, K.Y., M.C. Tu, and G.S. Shang. 2002. The Transition Kingdom - Guidebook of the Amphibians and Reptiles of Taiwan. SWAN, Taipei (in Chinese).

Luo, L.G., G.H. Ding, and X. Ji. 2010. Income breeding and temperature-induced plasticity in reproductive traits in lizards. The Journal of Experimental Biology 213: 2073-2078.

Norval, G. and J.J. Mao. 2008. A record of Takydromus kuehnei as prey of Sibynophis chinensis chinensis in a secondary forest in Chiayi County, Taiwan. Sauria 30: $51-54$.

Norval, G., S.C. Huang, and J.J. Mao. 2007. A description of Formosan Grass Lizard (Takydromus formosanus) clutches from a Betelnut Palm plantation in Chiayi County, Taiwan. Sauria 29: 46-48.

Shang, G.S. and S.L. Lin. 2001. Natural Portraits of the Lizards of Taiwan. Big Trees Publishers, Taipei (in Chinese).

Telford, S.R. 1969. The ovarian cycle, reproductive potential, and structure in a population of the Japanese lacertid Takydromus tachydromoides. Copeia 1969: $548-567$. 